\title{
Nephrotoxicity of Nickel Nanoparticles in Rat: Effect of Different Doses
}

\author{
Shang Z. Abdulqadir ${ }^{1}$ and Falah M. Aziz ${ }^{1}$
}

\begin{abstract}
The present investigation was dealt with the study of the effect of different doses of Nickel nanoparticles (NiNPs) (particle size equal to $20 \mathrm{~nm})$ on the kidney of rat. Intraperitoneal administration of NiNPs $(0,5,10,20,50$ and $100 \mathrm{mg} / \mathrm{kg}$ bw) was achieved for 28 days and the kidney samples were homogenized to estimate the level of malondialdehyde (MDA) as lipid peroxidation marker while creatinine was estimated in serum as well as studying the histopathological changes. Significant dose dependent elevation of MDA in kidney homogenate and a significant dose dependent increase of serum creatinine were detected. The most histopathological feature was the appearance of inflammatory aggregations of infiltrated leucocytes.
\end{abstract}

Keywords-Nickel nanoparticles, nephrotoxicity, MDA.

\section{INTRODUCTION}

Renal toxicity is one of the major concerns in preclinical toxicology studies. Renal toxicity can be a result of hemodynamic changes, direct injury to cells and tissue, inflammatory tissue injury, and/or obstruction of renal excretion. The kidney has important roles in plasma filtration and maintenance of metabolic homeostasis. Toxic effects on the kidney as a result of environmental toxicants can impair these kidney roles and induce changes in kidney function and structure [1].

Nanoparticles (NPs) are any materials or structures that have at dimension at $1-100 \mathrm{~nm}$ range [2]. With the development of nanotechnology, public concerns about the biological effects of NPs on the environment and human health have been raised [3]. NiNPs are widely used in hydrogen storages, chemical catalysts, ceramic capacitors, sensor and conductive paints, and nanomedicine over the past decade [4].

Another important sources of $\mathrm{Ni}$ particles uses which may bring them into environment are oil refineries and dust which may carry them [5]. Welding may generate a complex aerosol of incidental nanoparticles and cytotoxic metals, including nickel particles [6].

The majority of the published studies have assessed the cytotoxicity and genotoxicity of NPs on stable cell lines and in little degrees on laboratory animals. No doubt, in vitro experiments feature a number of advantages, in particular, relating to analysis of primary mechanisms of toxicity.

Shang Z. Abdulqadir and Falah M. Aziz both are with the Salahaddin University, Iraq
However, any extrapolation of such experimental results to the whole mammalian organism is associated with a number of uncertainties and assumptions. Moreover, some important aspects of toxicology (in particular, toxicokinetics, doseresponse relationships on organ and systemic levels, and the functioning of protective mechanisms) can generally be obtained only through experiments on a whole mammalian organism [7].

Previous studies had confirmed that Ni NPs might be more toxic than their fine particles even they may be more carcinogenic [8]-[9].

The major target organs for $\mathrm{Ni}$ compounds include liver, kidney, brain, lung and testes [10]. .Ni was found to cause neurotoxicity, hepatotoxicity, nephrotoxicity, gene toxicity, reproductive toxicity [11]-[12]-[13]-[14].

The main mechanism suggested for the cell damage induced by NiNPs is by causing of ROS elevation [15] which is considered to be a key mechanism for the cytotoxicity of nanomaterials [16]-[17]. NiNPs was also found to induce oxidative DNA damage [18], inflammation, cell degeneration [19], cell cycle arrest [20]. cytogenetic alterations, oxidative stress and apoptosis [21].

The increased production of $\mathrm{Ni}$-containing nanoparticles necessitates a thorough assessment of their toxicological properties [22].

The present investigation was aimed to focus more histological and cytological light on the nephrotoxicity of NiNPs using different doses of $20 \mathrm{~nm}$ size nickel nanoparticles.

\section{MATERIALS AND METHODS}

\section{A. Materials}

Powdered 20nm nickel nanoparticles (NiNPs) were purchased from Sigma-Aldrich Co. (St Louis, MO, USA). All other chemicals were of laboratory grade and were used as received. All other necessary reagents were of analytical grade and were purchased from Sigma-Aldrich Co.

\section{B. Preparation and Ultrasonication of NiNPs}

Five different doses of NiNPs solutions were prepared in normal saline $(5,10,20,50$ and $100 \mathrm{mg} / \mathrm{kg}$ body weight $) \mathrm{m} / \mathrm{mL}$ A stock suspension of NiNPs was prepared in normal saline $(10 \mathrm{mg} \backslash \mathrm{mL})$ by sonication for $30 \mathrm{sec}$ in ultrasonic homogenizer (model 150VT, manufactured by biologica, Ink, Manassas, Virginia, USA), the particle suspensions were kept on ice for $15 \mathrm{sec}$ and sonicated again on ice for a total of $3 \mathrm{~min}$ at power 
of $400 \mathrm{~W}$. The NiNPs is vibrated for $2 \mathrm{~min}$, immediately prior to the process of injection.

\section{Experimental Design}

Randomly 60 healthy mature female albino rats (Rattus norvegicus) weighing $200-220 \mathrm{gm}$ were divided into six groups, in group 1 (control) rats were injected intrapertoneally (i.p.) with normal saline, whereas the groups 2,3,4,5 and 6 (NiNPs-injected rats) were i.p. injected with ultrasonicated NiNPs $(5,10,20,50$ and $100 \mathrm{mg} / \mathrm{kg}$ body weight respectively). Injection of all groups were achieved for two days per a week for 28 days. At the end of administration, rats were sacrificed, and the kidney samples were removed for histological and biochemical studies.

\section{D.Preparation of Kidney Homogenate}

Kidney pieces of each rat were washed in ice-cold normal saline solution then homogenized in $20 \mathrm{mM}$ phosphate buffer $(\mathrm{pH}=7.4$; tissue/ buffer ratio, $1 / 10 \mathrm{w} / \mathrm{v})$ by handheld glass homogenizer. Kidney homogenates were centrifuged at 4,000 $\mathrm{g}$ at $4^{\circ} \mathrm{C}$ for 10 minutes (Beckman J2-21).The supernatants were collected and stored at $-80 \mathrm{C}$ until assayed.

\section{E. Determination of Kidney Homogenate Malondialdehyde} (MDA):

MDA level was estimated according to method of Kartha and Krishnamurthy [23]. One $\mathrm{ml}$ of the kidney homogenates were added to $20 \%$ trichloroacetic acid . After centrifugation for 10 minutes, $2 \mathrm{mls}$ of supernatant were taken in a test tube, $2 \mathrm{mls}$ of $0.7 \%$ thiobarbituric acid were added to each tube and kept in boiling water bath for 20 minutes. The development of pink color was measured at $535 \mathrm{~nm}$ and the value of MDA concentration was expressed by MDA(nmol/g of tissue).

\section{F. Blood Collection}

At the end of the treatment period, blood samples were collected from all anesthetized rats of six groups through cardiac puncture in which the collected blood samples were immediately placed into gel tube for serum collection, later were centrifuged (Hettich D-78532/Germany) at $3000 \mathrm{rpm}$ for 15 minutes .The sera were stored at $-80 \mathrm{C}$ (Sanyo - Ultra Low Temperature, Japan) for creatinine estimation.

\section{G.Estimation of Creatinine}

Serum creatinine was determined spectrophotometrically (Cobas c311 Hitachi-Japan) using BIOLABO kit(France) . Creatinine reacts with picrate to form a colored complex in alkaline solution; the intensity of the color is proportional to the concentration of the creatinine. Absorbency of samples were read at $500 \mathrm{~nm}$.

\section{H.Histological Techniques}

kidney pieces were fixed in $10 \%$ buffered formaldehyde for 24 hours, followed by dehydration using a series of ethanol in ascending concentrations $(50 \%, 70 \%, 95 \%$ and $100 \%)$, then immersed in xylene, infiltrated embedded in paraffin wax. Five micrometer thick sections were obtained using rotary microtome (Bright, MIC) and stained by hematoxylin and eosin (H\&E) [24].

\section{Electron Microscopy}

Scanning electron microscopy was used for photographing the NiNPs after coating with platinum.

\section{J. Statistical Analysis}

All data are expressed as means \pm standard error $(\mathrm{M} \pm \mathrm{SE})$ and statistical analysis was carried out using statistically available software (SPSS version 22). One-way analysis of variance (ANOVA) was performed to test for significance of a treatment effect followed by Duncan's multiple range comparison tests for comparisons between the groups. P values $\leq 0.05$ were considered significant..

\section{RESULTS AND DISCUSSION}

The present investigation which dealt with the study of the effect of different doses of 20nm sized NiNPs (as appeared in Fig 1A viewed under scanning electron microscopy) on the kidney of rats, revealed a significant $(\leq 0.05)$ dose dependent increase in the malondialdehyde level (Fig 1B). is the final product of lipid peroxidation process [25] and is still routinely used as a marker for oxidative stress induced by different toxicants [26]. Several works conclusions have agreed about the mechanism of NiNPs in inducing the toxicity in vivo [19] and in vitro [22]-[18] which is the inducting oxidative stress through elevating the levels of reactive oxygen species (ROS).

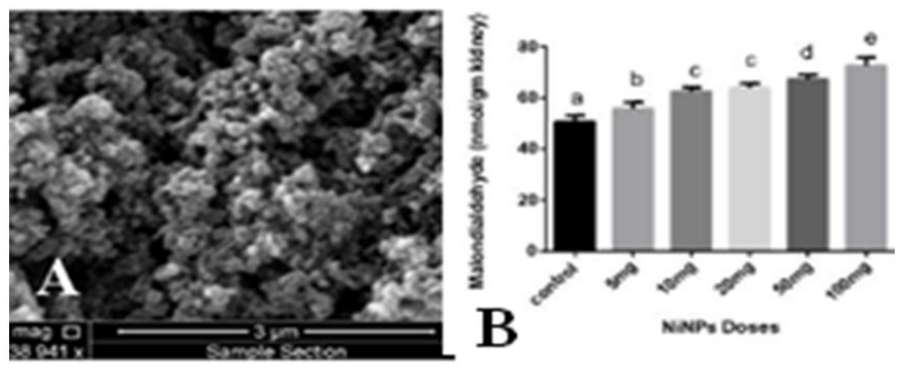

Fig.1: A) Nickel nanoparticles (NiNPs) as viewed by scanning electron microscopy, B) MDA in the kidney homogenate of rats exposed to different doses of NiNPs.

In the present investigation, NiNPs have caused a significant $(\mathrm{P} \leq 0.05)$ dose dependent elevation of serum creatinine in comparison to the control (Fig.2). This may be related to the NiNPs accumulated in the renal tissues, that enhanced the partial or full renal failure leading to decrease the efficiency of the kidney, and in turn hinder and/or disturb the excretion of the two metabolites from the circulation via the urine [27].

The high levels of creatinine could reflect the abnormal glomerular filtration rate due to the metals accumulation in kidney [28]. Among literatures, the results with respect to creatinine levels in response to NiNPs exposure were conflicting. In acute intravenous injection, no changes in crea- 


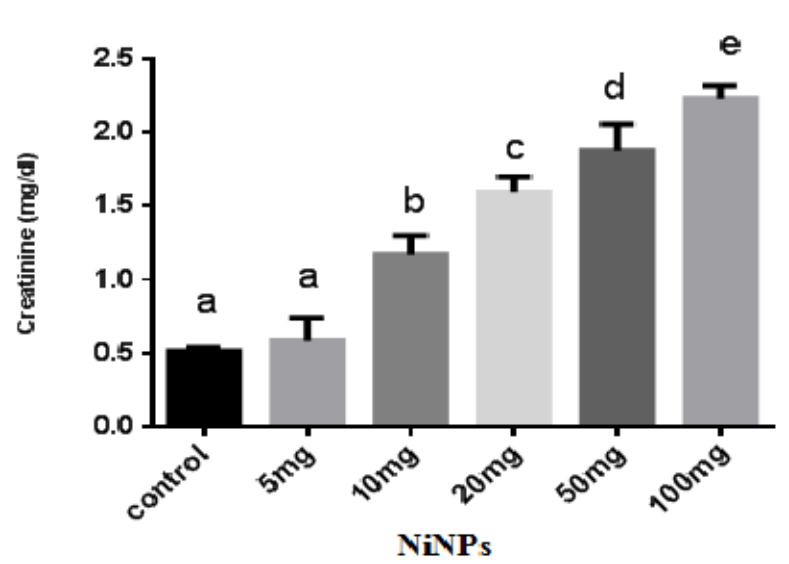

Fig. 2: Serum creatinine at different doses of NiNPs

-tinine level was observed [8], whereas after 3, 7, 14 and 28 days post-injection of NiNP, levels of creatinine were significantly higher than the corresponding controls, and positively correlated with the experimental periods [8].Recently, rats were exposed to acute ( 125,250 , and $500 \mathrm{mg} / \mathrm{kg}$ ) doses of NiO NPs in female Wistar rats and many nephritic biochemical changes have been recorded[29].

Histologically, the present investigation showed several changes in the structure of the kidney and the most important features were the appearance of inflammatory infiltrated leucocytes (Fig.3) in approximately dose dependent pattern. The inflammatory cells which were mainly lymphocytes were located in the interstitial spaces between the kidney tubules, around blood vessels and around the glomerului (Fig.4). Other morphological changes included dilatation of the kidney tubules and degeneration of kidney tubules epithelial cells. Recently, NiONPs were taken up by kidney cell line and induced dose-dependent DNA damage and apoptotic/nephritic effects[18]. Reference [30] studied the effects intraperitoneal injection and aspiration effects of nickel nanowire with diameter of $200 \mathrm{~nm}$ and length of more than $20 \mathrm{~lm}$ in mice and found out that they caused strong inflammation in mouse peritoneal cavity and lung respectively, in a dose-dependent manner. Nanoparticles were also found to up-regulate the transcription of various pro-inflammatory genes, including tumor necrosis factor- $\alpha$ and IL (interleukins)-1, IL-6 and IL-8, by activating nuclear factor-kappa $\mathrm{B}(\mathrm{NF}-\mathrm{kB})$ signaling. These sequential molecular and cellular events are known to cause oxidative stress, followed by severe cellular genotoxicity and then programmed cell death[31]. Those previous results that concerning the effect of nanoparticles on interleukins may explain the present nephrotoxic results such as inflammation. Dose dependent nephrotoxicity effect of NiNPs may be related to the dose dependent accumulation of these particles in the kidney throughout the 28 days of exposure [32].
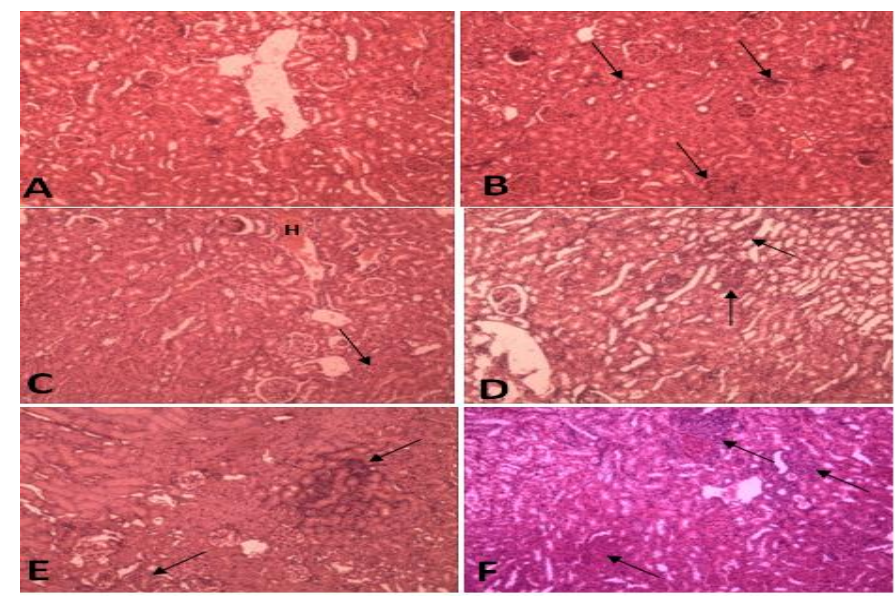

Fig.3 Sections through the kidney of different doses of NiNPs treated rats showing dose dependent nephrotoxic effect through the appearance of inflammatory infiltrated leucocytes aggregations (arrows) in the interstitial spaces between kidney tubules, $(\mathrm{H})$ : Hemorrhage, A) control group, B) $5 \mathrm{mg} / \mathrm{kg}$, C) $10 \mathrm{mg} / \mathrm{kg}$, D) $20 \mathrm{mg} / \mathrm{kg}$, E) $50 \mathrm{mg} / \mathrm{kg}$, F) $100 \mathrm{mg} / \mathrm{kg}$


Fig.4: Sections through the kidney of different doses of NiNPs treated rats showing dose dependent nephrotoxic effect through the appearance of inflammatory infiltrated leucocytes (arrows) in the interstitial spaces between kidney tubules, A) control group, B) $5 \mathrm{mg} / \mathrm{kg}$, C) $10 \mathrm{mg} / \mathrm{kg}$, D) $20 \mathrm{mg} / \mathrm{kg}$, E) $50 \mathrm{mg} / \mathrm{kg}$, F) $100 \mathrm{mg} / \mathrm{kg}$

\section{REFERENCES}

[1] F. Palm, and L. Nordquist, "Renal oxidative stress, oxygenation, and hypertension," Am. J. Physiol.-Regul. Integr. Comp. Physiol., vol. 301 (5), pp. R1229-R1241, November 2011. https://doi.org/10.1152/ajpregu.00720.2010

[2] I. Khana, Khalid B, Saeed, and I. Khan. "Nanoparticles: Properties, applications and toxicities," King Saud Univ. Arab J. Chem, submitted for publication , May 2017.

[3] K. Savolainen, H. Alenius, H. Norppa, L. Pylkkänen, T. Tuomi, and K. Kasper, "Risk assessment of engineered nanomaterials and nanotechnologies-a review," Toxicol. Vol. 269, (2-3), pp. 92-104, March 2010. https://doi.org/10.1016/j.tox.2010.01.013

[4] Q. Zhang, Y. Kusaka, K. Sato, K. Nakakuki, N. Kohyama, and K. Donaldson "Differences in the extent of inflammation caused by intratracheal exposure to three ultrafine metals: role of free radicals," $J$ Toxicol Environ Health A. vol. 53(6), pp. 423-438, March 1998. https://doi.org/10.1080/009841098159169 
[5] M. A. Al-Dabbas, G. A. Hussain, and M. E. Al-Jubory, "The Effect of North Refineries Company on Soil pollution of Baiji City-Iraq," Iraq. J. Sci. 55(3B), pp. 1319-1329, 2014.

[6] M. Shoeb, V. Kodali, B. Farris, B.M. Bishop, T. Meighan, R. Salmen,, et al. "Evaluation of the molecular mechanisms associated with cytotoxicity and inflammation after pulmonary exposure to different metal-rich welding particles," Nanotoxicol,

vol. 11(6), pp. 725-736, August 2017. doi: 10.1080/ 17435390. 2017. 1349200.

[7] I. A. Minigalieva, B. A. Katsnelson, L. I. Privalova, M. P. Sutunkova , V. B. Gurvich, V. Y. Shur, et al. "Attenuation of Combined Nickel(II) Oxide and Manganese(II, III) Oxide Nanoparticles' Adverse Effects with a Complex of Bioprotectors," Int. J. Mol. Sci. vol. 16, pp. 22555-22583, September 2015. doi:10.3390/ijms160922555G.

[8] R. Magaye, Q. Zhou, L. Bowman, B. Zou, G. Mao, J. Xu, et al. "Metallic nickel nanoparticles may exhibit higher carcinogenic potential than fine particles in JB6 cells," PloSone. 2014; 9(4):e92418. April 2014. doi: 1371/journal.pone .0092418

[9] B. A. Katsnelson, L. Privalova, M. P. Sutunkova, V. F. Gurvich, N. V. Loginova, I. A. Minigalieva, et al. "Some inferences from in vivo experiments with metal and metal oxide nanoparticles: the pulmonary phagocytosis response, subchronic systemic toxicity and genotoxicity, regulatory proposals, searching for bioprotectors (a self-overview) ,"Int.l. J. Nanomed.,10 pp. 3013-29, April 2015.

[10] L. Pari, and A. Prasath, "Efficacy of caffeic acid in preventing nickel induced oxidative damage in liver of rats," Chem-Biol Interact 173(2), pp.77-83, May 2008.

[11] K. H. Gathwan, I.H. T. Al-Karkhi, and E. A. Jaffar Al-Mulla, "Hepatic toxicity of nickel chloride in mice," Res. Chem. Intermed., 39(6), pp. 2537-42, July 2012.

[12] Z. Forgacs, P. Massanyi, N. Lukac, and Z. Somosy, "Reproductive toxicology of nickel-Review," J. Environ. Sci. Health A., vol. 47(9), pp. 1249-1260, November 2012. https://doi.org/10.1080/10934529.2012.672114

[13] C. Y. Chen, T. K. Lin, Y. C. Chang, Y. F. Wang, H. W. Shyu, K. H. Lin, et al. "Nickel (II)-induced oxidative stress, apoptosis, G2/M arrest, and genotoxicity in normal rat kidney cells," J. Toxicol. Environ. Health A, vol. 73(8), pp. 529-539, 2010. https://doi.org/10.1080/15287390903421250

[14] L. Kong, M. Tang, T. Zhang, D.Wang, K. Hu, W. Lu, et al. "Nickel Nanoparticles Exposure and Reproductive Toxicity in Healthy Adult Rats," Int J Mol Sci. vol. 15(11), pp. 21253-69 November 2014. doi: $10.3390 /$ ijms 151121253

[15] S. Yu, F. Liu, C. Wang, J. Zhang, A. Zhu, L. Zou, et al. "Role of oxidative stress in liver toxicity induced by nickel nanoparticles in rats," Mol Med Rep. 17(2) pp. 3133-9, February 2018. doi:10.3892/mmr.8226 to be published

[16] A. Nel, T. Xia, L. Mädler, and N. Li, "Toxic potential of materials at the nanolevel,". Sci. vol. 311, pp. 622-627, February 2006. https://doi.org/10.1126/science.1114397

[17] M. Meng, T. Xia, S. George, and A. E. Nel, "A predictive toxicological paradigm for the safety assessment of nanomaterials," ACS Nano. vol. 3(7), pp. 1620-1627, July 2009. https://doi.org/10.1021/nn9005973

[18] M. Abudayyak, E. Guzel, and G. Özhan, "Nickel Oxide Nanoparticles Induce Oxidative DNA Damage and Apoptosis in Kidney Cell Line (NRK-52E)," Biol Trace Elem Res. Vol 178 (1): pp. 98-104. July 2017.

[19] S. T. Razavipour, M. Behnammorshedi, R. Razavipour, and M. Ajdary "The toxic effect of nickel nanoparticles on oxidative stress and inflammatory markers," Biomed Res, vol. 26 (2): pp. 370-374, February 2015.

[20] C. Ma, M. Songb, Y. Zhangb, M. Yanb, M. and Zhanga, H. Bi, "Nickel nanowires induce cell cycle arrest and apoptosis by generation of reactive oxygen species in HeLa cells," Toxicol. Rep,vol. 1, pp. 114121, May 2014. https://doi.org/10.1016/j.toxrep.2014.04.008

[21] Q. Saquib, S. M. Attia, S. M. Ansari, A. Al-Salim, M. Faisal, A. A. Alatar, et al. "p53, MAPKAPK-2 and caspases regulate nickel oxide nanoparticles induce cell death and cytogenetic anomalies in rats," Int J Biol Macromol, vol. 105(pt 1), pp. 228-237, 2017.. doi: 10.1016/j.ijbiomac.
[22] S. Latvala, J. Hedberg, S. Di Bucchianico, L. Möller, I. Wallinder, K. Elihn, et al. "Nickel Release, ROS Generation and Toxicity of Ni and NiO Micro- and Nanoparticles," \PLosone. Vol 11(7), July 2016. DOI:10.1371 /journal. pone. 0159684

[23] V. N. Kartha, and S. Krishnamurthy, "Factors affecting in vitro lipid peroxidation of rat brain homogenate," Ind. J. physio. pharmacol., vol. 22(1): pp. 44-52, January-March 1977.

[24] J. D. Bancroft, and A. Stevens, "Histopathological Stains and Their Diagnostic Uses," Churchill Livingstone. Edinburgh, pp.134 - 137, 1975

[25] A. Ayala, M. Muñoz, and S. Argüelles "Lipid Peroxidation: Production, Metabolism, and Signaling Mechanisms of Malondialdehyde and 4Hydroxy-2-Nonena," Oxid Med Cell Longe Vol. 2014 May 2014 Article ID 360438, 31 pages http:// dx.doi. org/10.1155/2014/360438

[26] M. Khoubnasabjafari, K. Ansarin, and A. Jouyban, "Reliability of malondialdehyde as a biomarker of oxidative stress in psychological disorders," BioImpac, vol.5(3), pp. 123-127, July 2015. doi: 10.15171/bi.2015.20 https://doi.org/10.15171/bi.2015.20

[27] D. H. Kang, T. Nakagawa, L. Feng, S. Watanabe, L. Han, M. Mazzali, et al. "A role for uric acid in the progression of renal disease," Journal of the American Society of Nephrology, vol. 13(12): pp. 2888-2897. 2002 https://doi.org/10.1097/01.ASN.0000034910.58454.FD

[28] I. I. Abdel Aziz,. and B. M. Zabut, "Determination of blood indices of albino rats treated with aluminum chloride and investigation of antioxidant effects of vitamin E and C,". Egypt. J. Biol., vol. 13, pp. 1-7. July 2011.

[29] N. Dumala, B. Mangalampalli, S. K. Kamal and P. Grover, "Biochemical alterations induced by nickel oxide nanoparticles in female Wistar albino rats after acute oral exposure," Biomarkers, vol. 22(8), pp. 1-11, August 2017.

[30] C. A. Poland, F. Byrne, W.S. Cho, A. Prina-Mello, F.A Murphy, and G.L, Davies, "Length-dependent pathogenic effects of nickel nanowires in the lungs and the peritoneal cavity," Nanotoxicology vol. 6, pp. 899911, December 2012. doi:10.3109/17435390. 2011.626535.

[31] P. Khanna, C. Ong, B. Bay and G. Baeg "Nanotoxicity: An Interplay of Oxidative Stress, Inflammation and Cell Death," Nanomat. Basel, vol. 5(3), pp. 1163-1180, June 2015. https://doi.org/10.3390/nano5031163

[32] G. Morsy, and N. Elkon, "Bioaccumulation of nickel nanopowder and evaluation of possible toxicity in male albino rats," Ey. J. Zool., vol. 61, pp. 275-299, June 2014. https://doi.org/10.12816/0005519 